\section{Heavy Duty Camera Bellows for Digital Imaging}

Theodore M. Clarke, Case Corporation Tech Center

My previous article on digital imaging in the April 1997 issue of Microscopy Today noted the use of an Olympus Auto Bellows with a Kodak MegaPlus 1.6i/AB CCD camera for digital photomacrographs equivalent to 4 $x 5$ Polaroid prints with magnifications between $10 \mathrm{X}$ and $50 \mathrm{X}$. The purpose of this article is to document the high resolution obtainable with the bellows system and the design modifications needed to eliminate the plastic dovetail inserts of the Olympus Auto Bellows, which are susceptible to delayed stress cracking when used with a heavier camera like the MegaPlus. Total failure of the dovetail inserts could cause costly damage to the lens and the camera.

The readers may question the use of a bellows and macro lenses when a stereo microscope is commonly used for photomacrography in the $6 \mathrm{X}-50 \mathrm{X}$ magnification range. Our high resolution Zeiss Stemi SV 11 stereo microscope is located in the SEM laboratory, where it is most needed. The MegaPlus camera is located in the metallography laboratory with a metallurgical microscope on one side of the PC and a copy stand on the other side. The copy stand is used to document large features with the MegaPlus camera and a $28 \mathrm{~mm}$ focal length lens. A $60 \mathrm{~mm}$ focal length Micro Nikkor lens is used for most of the photomacrography, up to a magnification equivalent to a $10 \times 4 \times 5$ Polaroid print. $A$ bellows is used with Zeiss Luminar lenses, $100 \mathrm{~mm}$ and $63 \mathrm{~mm}$ focal length, to cover the $10 \mathrm{X}$ to $50 \mathrm{X}$ magnification range. This permits all of the photography and photomacrography of a specimen to be done in a single session without the need for a stereo microscope dedicated for use with the digital imaging system. Mea-

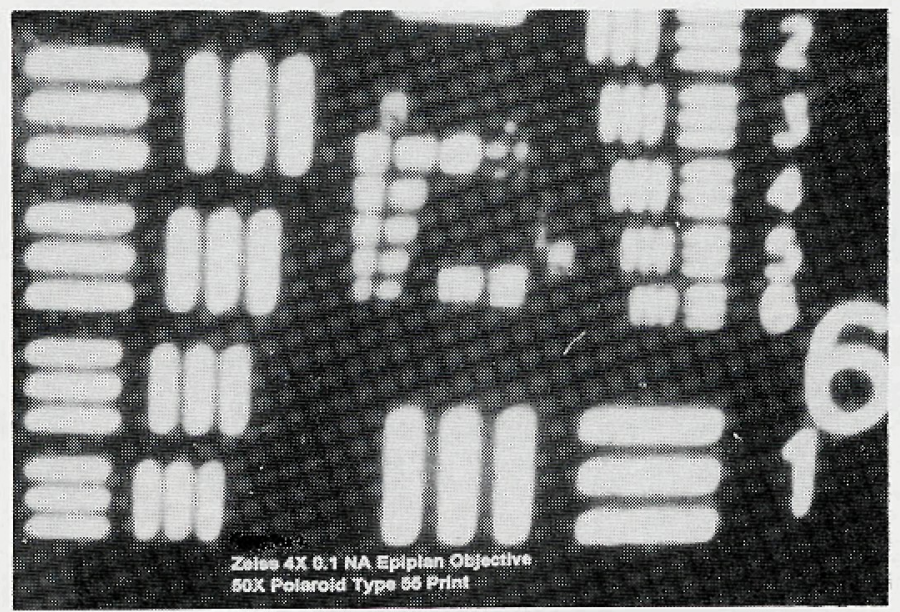

Figure 1

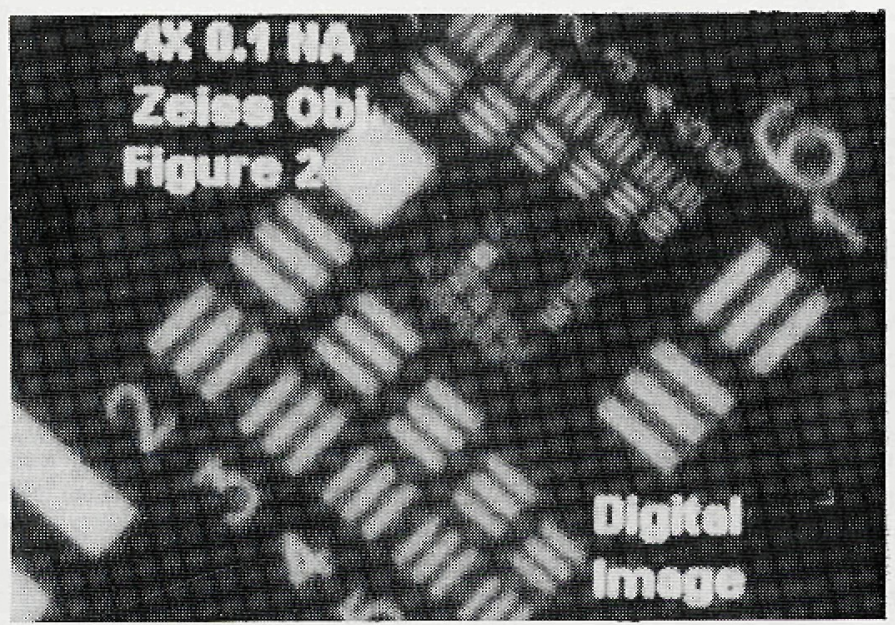

Figure 2 sured resolution test data for macro lenses used on camera bellows is apparently known only by the manufacturers of these lenses because this information is not requested by the users of these lenses. Consequently, appropriate quantitative resolution test standards and results are not readily available for stereo microscopes and the lower power microscope objectives. The European manufacturers of stereo microscopes will furnish resolution performance data when it is requested, but do not use this information to their advantage when competing with less capable Asian microscopes. Fortunately, there is a resolution test pattern marketed by Applied Image Inc. of Rochester, New York, for evaluating stereo microscope and macro lens resolution. ${ }^{1}$ This chrome on glass pattern is used in our laboratory. Although the pattern can be used with either reflected or transmitted bright field illumination, diffuse transillumination was chosen for the tests in order to maximize the image contrast. Theoretical resolution is calculated from classical equations. ${ }^{2,3}$

\section{Object Resolution in lines/mm $=3000 \mathrm{NA}=\frac{1500 \text { Camera Mag }}{\text { f/number (Camera Mag+1) }}$}

The resolution pattern recorded at 50X with a Zeiss Ultraphot and 0.1 NA objective on $4 \times 5$ Polaroid Type 55 P/N film was chosen as a basis for comparison with a digital photomicrograph obtained with the same objective and digital photomacrographs recorded at $5 \mathrm{X}$ camera magnification. The test patterns were oriented at about $45^{\circ}$ to the $C C D$ axes to obtain maximum resolution and avoid alias line effects, caused by undersampling when the special frequency in the optical image formed on the sensor approaches the pixel spacing of the $\mathrm{CCD}$. The spacing of rows of pixels touching on the corner diagonals is 707 of the basic spacing of the orthogonal array of square pixels and presumably related to the higher measured resolution with test pattern lines oriented at $45^{\circ}$. The finest

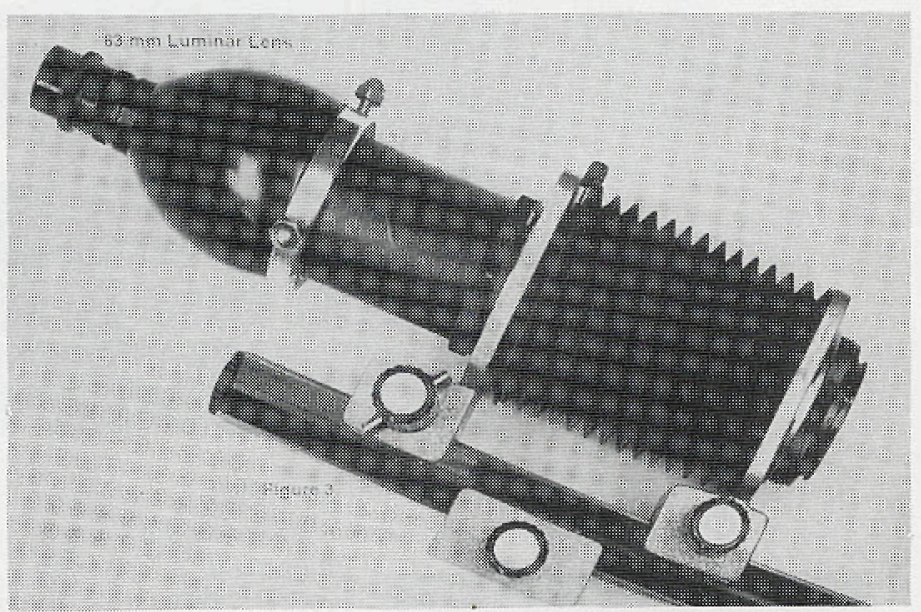

Figure 3

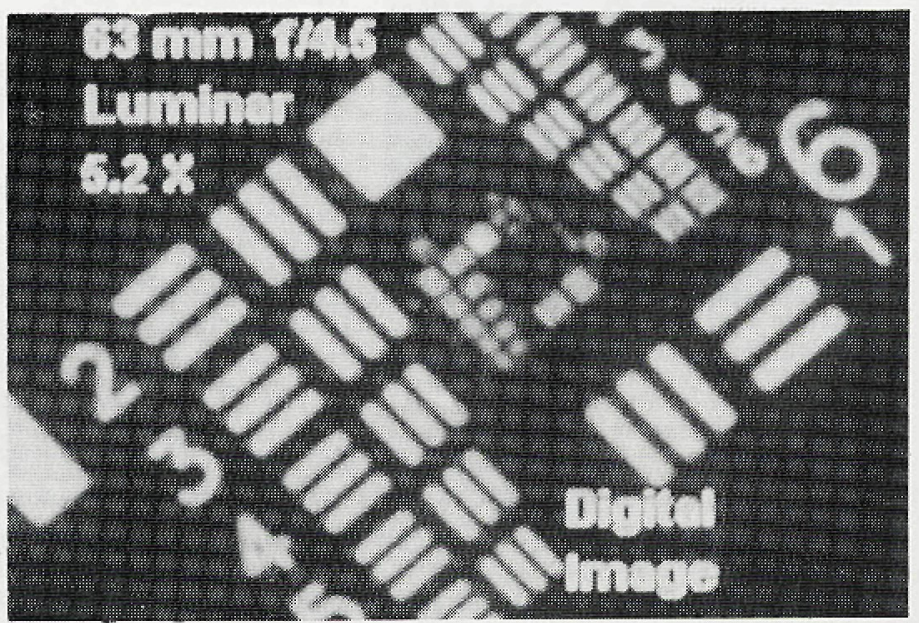

Figure 4 
pattern resolved on the 50X Polaroid is 287 lines per $\mathrm{mm}$ (Group \#8 and Element \#2), see Figure 1. The theoretical resolution of a $0.10 \mathrm{NA}$ objective is 300 lines $/ \mathrm{mm}$. The finest pattern just resolved with this objective using the Kodak MegaPlus camera is 256 lines/mm (Group \#8 and Element \#1), see Figure 2.

High magnification photomacrography in the laboratory was previously done with a Zeiss Ultraphot II microscope using Luminar lenses. Fortunately, these lenses were optically corrected for $35 \mathrm{~mm}$ format and lower camera magnifications than when used on the Ultraphot with its 1 meter bellows draw and $4 \times 5$ camera back. My personal camera equipment is based upon the Olympus Camera system. The Olympus Auto Bellows was a good candidate for use with the MegaPlus camera and the Zeiss Luminar lenses in the Case Materials Engineering Laboratory. I made an extension tube in my home machine shop to couple the Zeiss lens holders to one of my Olympus Auto Bellows and modified the camera mount from $O M$ to Nikon $F$ mount. The resulting bellows system is shown in Figure 3. A stereo microscope eyepiece in a holder adjusted to be parfocal with the camera is used to locate the field on the object for digital imaging with the camera mounted in place of the eyepiece holder. The maximum magnification of the $63 \mathrm{~mm}$ Luminar on ths bellows is $5.2 X$, which is less than a factor of two of the optimum magnification of $3 X$ for this lens. The theoretical resolution of this lens at $5.2 X$ and $f / 4.5$ is 280 lines $/ \mathrm{mm}$. The finest pattern resolved in a digital photomacrograph with this lens is 256 lines $/ \mathrm{mm}$ (Group \#8 and Element \#1), see Figure 4. This is also the finest pattern resolved with the $1 X$ objective of the Zeiss SV 11 stereo microscope using $25 X$ Zeiss eyepieces.

The Olympus bellows was used for about a year when cracks were detected in the plastic dovetail inserts, see Figure 5 . These plastic inserts were eliminated in my homemade heavy duty bellows shown in Figures 6 and 7 . The rack and pinion focusing and auto aperture closing linkage of the original Olympus design

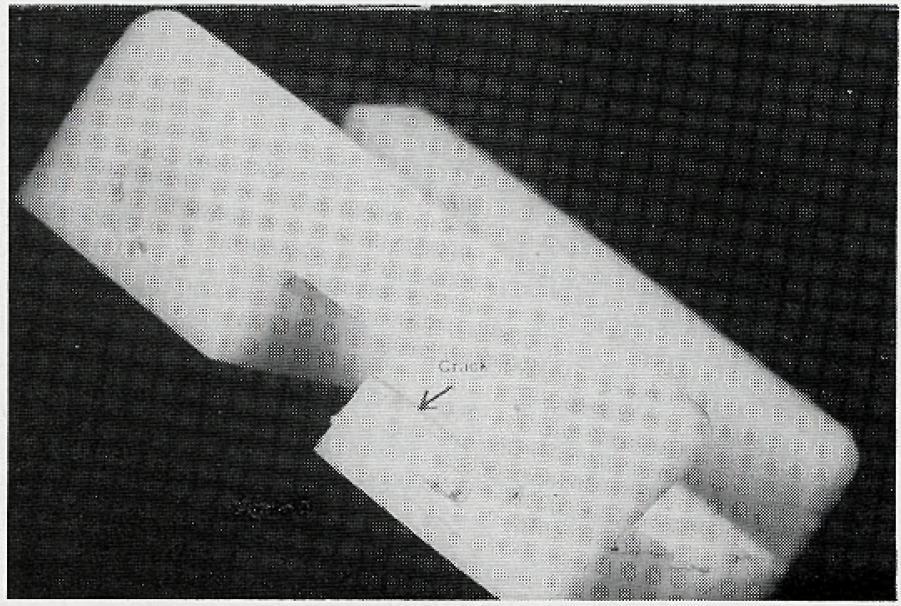

Figure 5

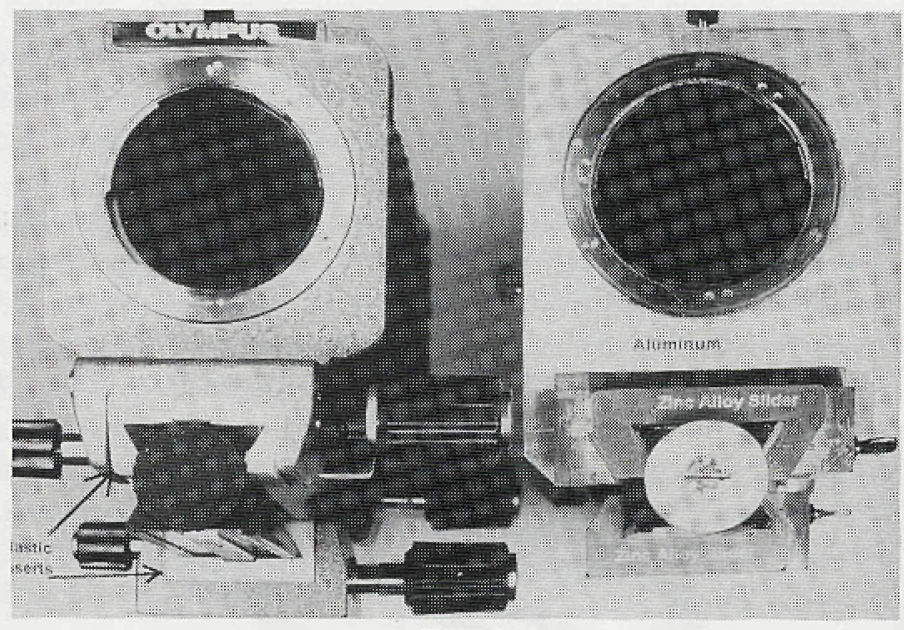

Figure 6 were eliminated in the heavy duty version. The female dovetail slides were machined from continuous cast zinc aluminum alloy ZA12 bar stock furnished by Zincaloy $I n{ }^{4}{ }^{4}$ This bearing alloy was very easily machined to high precision using high speed steel tool bits. The resulting bellows is much more rigid and very easy to use with the MegaPlus camera. No lubrication is used on the bellows rail, yet the movement is silky smooth. The lens and camera boards were machined from aluminum plate. These separate boards could be integrated into single plece castings of the ZA12 alloy for production quantities using low cost graphite molds. The $f / 2.838 \mathrm{~mm}$ Olympus Zuiko macro lens is capable of higher resolution than the f/4.5 $63 \mathrm{~mm}$ Luminar lens, but with the disadvantage of shorter working distance. The fine focusing ring on the Olympus lens is an advantage. The Olympus lens resolved 287 lines/mm (Group \#8 and Element \#2) at f/4 and 323 lines/mm (Group \#8 and Element \#3) at f/2.8, see Figure 8. The corresponding theoretical resolution limits are 300 lines $/ \mathrm{mm}$ and $430 \mathrm{lines} / \mathrm{mm}$. This later value exceeds the resolution of the $\mathrm{CCD}$ array. Laboratories fortunate enough to own a Wild M400 Macroscope should be able to match the digital imaging performance of the bellows system. Other laboratories with limited capital budgets will hopefully find the information in this article helpful for maximizing the return on their investment in high resolution digital imaging.

\section{References}

1. Applied Image Inc., 1653 East Main St., Rochester, N. Y. 14609 USA.

2. Clarke, T. M., "Resolution Considerations for Photomacrography and Photomicroscopy"; Microscopy Today May 96, 10-11.

3. Schillaber, C. P., "Photomicrography in Theory and Practice", John Wiley \& Sons, Inc. (1944).

4. Zincaloy Inc. 1385 Bonhill Rd., Mississauga, Ontario, Canada L5T 1 M1.

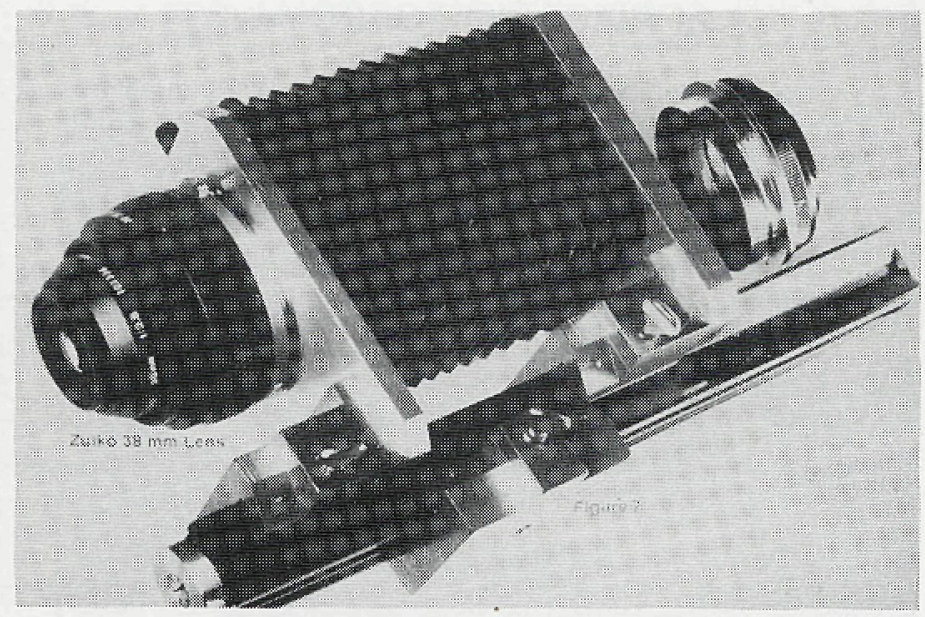

Figure 7

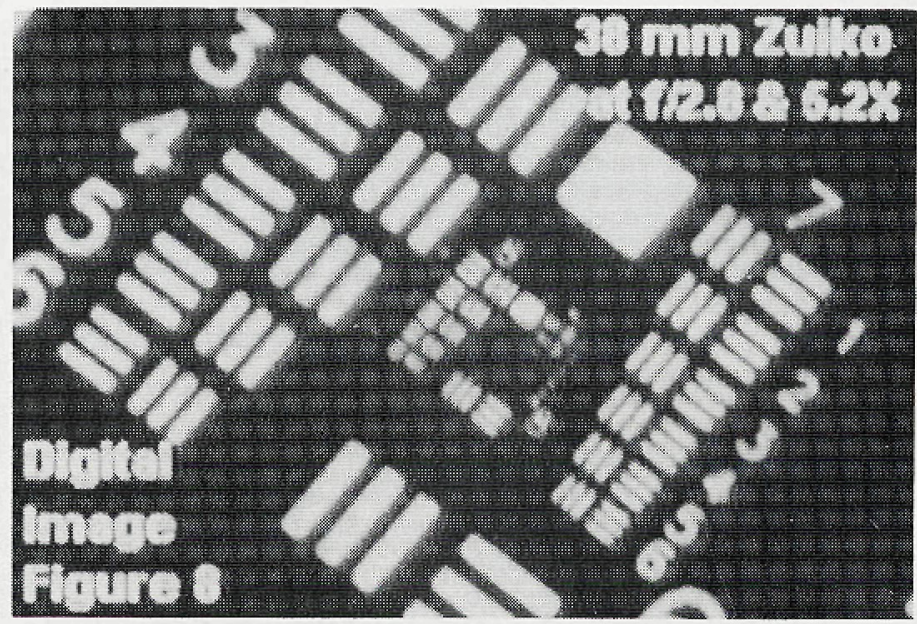

Figure 8 